\title{
Histone deacetylase inhibitors and aspirin interact synergistically to induce cell death in ovarian cancer cells
}

\author{
JÜRGEN SONNEMANN ${ }^{1}$, ISABEL HÜLS ${ }^{2}$, MICHAEL SIGLER ${ }^{2}$, CHITHRA D. PALANI ${ }^{2}$, \\ LE THI THU HONG ${ }^{3}$, UWE VÖLKER ${ }^{3}$, HEYO K. KROEMER $^{4}$ and JAMES F. BECK ${ }^{1}$ \\ ${ }^{1}$ University Children's Hospital Jena, Jena; ${ }^{2}$ Center of Pharmacology and Experimental Therapeutics, ${ }^{3}$ Laboratory for \\ Functional Genomics, ${ }^{4}$ Department of Pharmacology, Ernst Moritz Arndt University, Greifswald, Germany
}

Received November 28, 2007; Accepted March 3, 2008

\begin{abstract}
Histone deacetylase inhibitors (HDIs) as well as non-steroidal anti-inflammatory drugs including aspirin show promise as antineoplastic agents. The treatment with both HDIs and aspirin can result in hyperacetylation of proteins. In this study, we investigated whether HDIs and aspirin interacted in inducing anticancer activity and histone acetylation. We found that the HDIs, suberoylanilide hydroxamic acid and sodium butyrate, and aspirin cooperated to induce cell death in the ovarian cancer cell line, A2780. The effect was synergistic, as evidenced by CI-isobologram analysis. However, aspirin had no effect on histone acetylation, neither in the absence nor presence of HDIs. To gain insight into the mechanism underlying the synergistic action of HDIs and aspirin, we employed the deacetylated metabolite of aspirin, salicylic acid, and the cyclooxygenase-1- and -2-selective inhibitors, SC-560 and NS-398, respectively. We found that HDIs and salicylic acid interacted synergistically, albeit less efficiently than HDIs and aspirin, to induce cancer cell death, suggesting that the acetyl and the salicyl moiety contributed to the cooperative interaction of aspirin with HDIs. SC-560 and NS-398 had little effect both when applied alone or in conjunction with HDIs, indicating that the combinatorial effect of HDIs and aspirin was not the result of cyclo-oxygenase inhibition. In conclusion, our study demonstrates that HDIs and aspirin synergize to induce cancer cell death and, thus, provides a rationale for a more in-depth exploration into the potential of combining HDIs and aspirin as a strategy for anticancer therapy.
\end{abstract}

\section{Introduction}

Histone deacetylase inhibitors (HDIs) are emerging as a promising new class of relatively specific anticancer agents.

Correspondence to: Dr James F. Beck, Klinik für Kinder und Jugendmedizin, Friedrich-Schiller-Universität Jena, Kochstr. 2, D-07743 Jena, Germany

E-mail: james.beck@med.uni-jena.de

Key words: ovarian cancer, histone deacetylase inhibitors, aspirin, salicylic acid, SC-560, NS-398
Many in vitro and animal studies have demonstrated that they can induce differentiation and apoptosis, inhibit cell proliferation, and exert immune stimulatory and antiangiogenic activities in tumor cells $(1,2)$. Additionally, HDIs have been shown to enhance the anti-neoplastic efficiency of other therapeutic regimens, such as ionizing radiation or chemotherapy. Early-phase clinical trials revealed that HDIs have anticancer activity in a variety of solid and hematological malignancies also in a clinical setting. HDIs function by inhibiting histone deacetylases, thus increasing the acetylation of lysine moieties in histones, in turn leading to an increase in transcriptionally active chromatin.

Likewise, aspirin and aspirin-like agents, referred to as non-steroidal anti-inflammatory drugs (NSAIDs), show promise as antineoplastic drugs. Numerous studies suggest that the use of NSAIDs, primarily aspirin, decreases the risk of several malignancies (3-5), including ovarian cancer (6). NSAIDs primarily function by inhibiting cyclooxygenase (COX) activity, thereby preventing the biosynthesis of prostaglandins (7), but also other modes of action may account for their effects (8). Although there is mounting evidence that their antineoplastic activity rests on their ability to promote apoptosis in tumor cells, their way of action is under debate; both COX-dependent and -independent cellular processes seem to be involved $(3,4,8)$.

Aspirin inhibits COX enzymes by the acetylation of a specific serine residue. In addition, it has been reported to acetylate other proteins, chiefly on lysine moieties (9). We, thus, hypothesized that aspirin may cooperate with HDIs in promoting histone acetylation and in exerting antitumor effects. We investigated whether HDIs belonging to two different structural classes, the hydroxamic acid derivative suberoylanilide hydroxamic acid (SAHA; vorinostat) and the short-chain fatty acid sodium butyrate $(\mathrm{NaB})$, and aspirin interacted synergistically to induce cell death in an ovarian cancer cell line. Ovarian cancer appeared to be particularly interesting, because i) treatment with NSAIDs including aspirin have been demonstrated in vitro to reduce tumor growth (10), ii) an ovarian cancer mouse model study suggests that addition of SAHA could potentially increase the efficacy of the standard chemotherapeutic agent paclitaxel (11), and iii) in a recent ex vivo study we showed that SAHA had efficient activity against patient-derived ovarian cancer cells, which displayed only marginal responsiveness to paclitaxel 
(12). In this study, we show that the combination of HDIs with aspirin produced synergistic cytotoxic effects in A2780 ovarian cancer cells, while the combination of HDIs with selective COX inhibitors had no effect.

\section{Materials and methods}

Reagents. SAHA and NS-398 were purchased from Alexis (Grünberg, Germany). NaB, aspirin, and salicylic acid were purchased from Sigma (Deisenhofen, Germany). SC-560 was purchased from Merck Biosciences (Schwalbach, Germany). Aspirin and salicylic acid were adjusted to $\mathrm{pH} 7.4$ with $\mathrm{NaOH}$.

Cell culture. A2780 ovarian carcinoma cells were maintained in RPMI-1640 medium supplemented with $10 \%$ fetal calf serum, $2 \mathrm{mM}$ L-glutamine, 100 units $/ \mathrm{ml}$ penicillin $\mathrm{G}$ sodium, and $100 \mu \mathrm{g} / \mathrm{ml}$ streptomycin sulfate (media and supplements were purchased from Biochrom, Berlin, Germany). Cells were cultivated at $37^{\circ} \mathrm{C}$ in a humidified $5 \% \mathrm{CO}_{2}$ incubator and routinely passaged when $90-95 \%$ confluent. Cell viability was determined by the trypan blue exclusion test. Cells were regularly inspected to be free of Mycoplasma with Mycoplasma detection reagents from Roche (Mannheim, Germany).

Treatment of cells. The cells were plated at $2 \times 10^{5}$ cells in 6-well plates and treated with SAHA or $\mathrm{NaB}$ for $1 \mathrm{~h}$ or left untreated before application of aspirin (or alternatively salicylic acid, SC-560, or NS-398). Aspirin was added directly to the culture medium containing HDIs without a medium change. Cells were then cultivated for another $48 \mathrm{~h}$.

Flow cytometric analysis of cell death. Cell death was assessed by determining the integrity of the cell membrane by cytofluorometric analysis of propidium iodide (PI) uptake. Cells were harvested after the indicated treatments, followed by a 5-min incubation in $2 \mu \mathrm{g} / \mathrm{ml} \mathrm{PI} \mathrm{(Sigma)} \mathrm{in} \mathrm{PBS} \mathrm{at} 4^{\circ} \mathrm{C}$ in the dark. PI uptake was measured by flow cytometry analysis on a Becton Dickinson (Heidelberg, Germany) FACSCalibur. In each sample, 10,000 cells were analyzed; data were gated to exclude debris. The results from the assays were analyzed by the combination index (CI) method according to Chou and Talalay (13) using Calcusyn software from Biosoft (Cambridge, UK). CI values $>1.1$ indicate antagonism, between 0.9 and 1.1 additivism, and values $<0.9$ synergism .

Flow cytometric analysis of mitochondrial transmembrane potential $\left(\Delta \psi_{m}\right) . \Delta \psi_{\mathrm{m}}$ was determined by assessing the accumulation of the cationic lipophilic fluorochrome $3,3^{\prime}$-dihexyloxacarbocyanine iodide $\left[\operatorname{DiOC}_{6}(3)\right]$ in the mitochondrial matrix. After the indicated treatments, cells were incubated with $50 \mathrm{nM} \mathrm{DiOC}_{6}(3)$ (Molecular Probes, Eugene, OR, USA) at $37^{\circ} \mathrm{C}$ for $30 \mathrm{~min}$ prior to harvesting. After washing, 10,000 cells were analyzed using a FACSCalibur. Data were gated to exclude debris.

Western blot analysis. Cell were lysed on ice for $15 \mathrm{~min}$ in $40 \mathrm{mM}$ Tris- $\mathrm{HCl}$ (pH 7.4), $150 \mathrm{mM} \mathrm{NaCl}, 1 \%$ Triton X-100, $0.5 \%$ sodium deoxycholate, and $0.1 \%$ SDS supplemented with a protease inhibitor cocktail (Roche) followed by brief
A

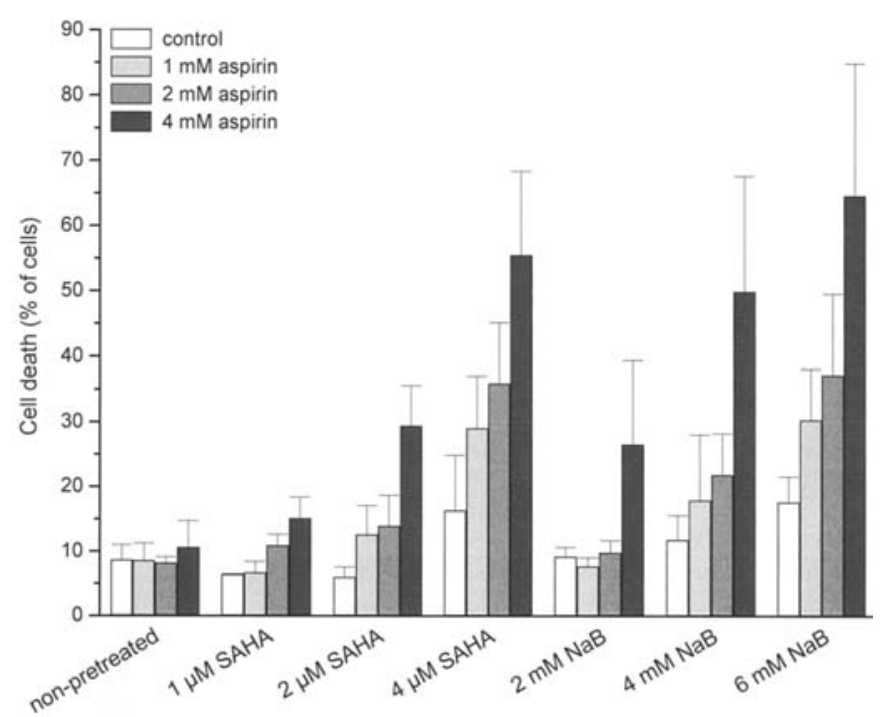

B

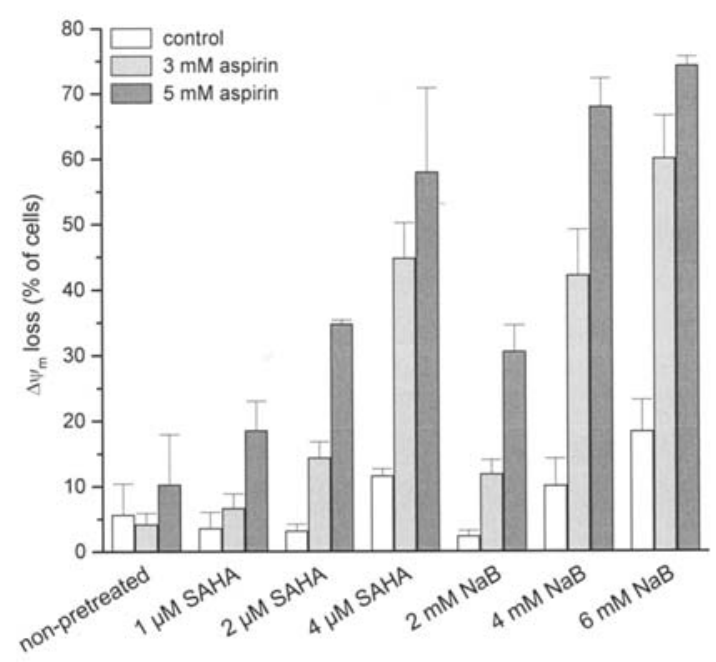

C
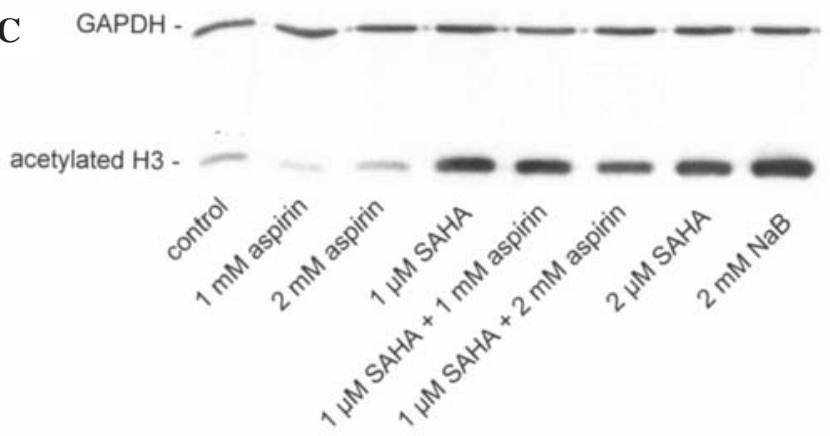

Figure 1. Induction of cell death in A2780 ovarian cancer cells by HDIs and aspirin. After 1-h treatment with SAHA or $\mathrm{NaB}$, cells were exposed to aspirin for another $48 \mathrm{~h}$. (A) Cell death was determined by flow cytometric analysis of PI uptake. (B) $\Delta \psi$ was assessed by flow cytometric analysis of DiOC $_{6}(3)$ staining. Means $\pm \mathrm{SD}$ of each three separate experiments are shown. (C) Immunoblotting for acetylated histone H3. After 1-h treatment with SAHA, cells were exposed to aspirin for $24 \mathrm{~h}$. Total protein $(30 \mu \mathrm{g})$ was separated by a $15 \%$ polyacrylamid gel.

sonification. Protein concentration was assayed using bicinchoninic acid (Pierce, Rockford, IL) according to the manufacturer's instructions. For immunoblotting, $30 \mu \mathrm{g}$ of 
Table I. Combination index values for SAHA plus aspirin.

\begin{tabular}{lcc}
\hline SAHA $(\mu \mathrm{M})$ & Aspirin $(\mathrm{mM})$ & CI \\
\hline 1.0 & 1.0 & 4.34 \\
1.0 & 2.0 & 0.65 \\
1.0 & 4.0 & 0.28 \\
2.0 & 1.0 & 0.63 \\
2.0 & 2.0 & 0.55 \\
2.0 & 4.0 & 0.14 \\
4.0 & 1.0 & 0.29 \\
4.0 & 2.0 & 0.19 \\
4.0 & 4.0 & 0.07 \\
\hline
\end{tabular}

Based on data from Fig. 1A, CI values were calculated using the CI-isobologram method of Chou and Talalay (13).

Table II. Combination index values for sodium butyrate plus aspirin.

\begin{tabular}{lcc}
\hline $\mathrm{NaB}(\mathrm{mM})$ & Aspirin $(\mathrm{mM})$ & $\mathrm{CI}$ \\
\hline 2.0 & 1.0 & 2.73 \\
2.0 & 2.0 & 1.44 \\
2.0 & 4.0 & 0.13 \\
4.0 & 1.0 & 0.58 \\
4.0 & 2.0 & 0.39 \\
4.0 & 4.0 & 0.06 \\
6.0 & 1.0 & 0.30 \\
6.0 & 2.0 & 0.18 \\
6.0 & 4.0 & 0.03 \\
\hline
\end{tabular}

Based on data from Fig. 1A, CI values were calculated using the CI-isobologram method of Chou and Talalay (13).

total cellular protein per lane were separated by standard SDS-PAGE on $10 \%$ gels and electrophoretically transferred to PVDF membranes (Millipore, Eschborn, Germany). After blocking in PBS containing 5\% dry milk and $0.05 \%$ Tween-20, acetylated histone $\mathrm{H} 3$ was immunodetected using rabbit anti-acetylated histone $\mathrm{H} 3$ (dilution 1:10,000; Upstate Biotechnology, Lake Placid, NY) polyclonal antibody. Even loading of protein was verified by detection of GAPDH using mouse anti-GAPDH monoclonal antibody (dilution 1:10,000; Biodesign International, Saco, ME). Peroxidase-conjugated goat anti-rabbit or anti-mouse IgGs (dilution 1:12,500; Dianova, Hamburg, Germany) followed by enhanced chemiluminescence (Amersham Biosciences, Freiburg, Germany) were used for detection.

\section{Results}

Effect of histone deacetylase inhibitors in combination with aspirin in A2780 ovarian cancer cells. To test a possible favorable cytotoxic interaction between HDIs and aspirin, we
Table III. Combination index values for SAHA plus salicylic acid.

\begin{tabular}{lcc}
\hline SAHA $(\mu \mathrm{M})$ & Salicylic acid $(\mathrm{mM})$ & CI \\
\hline 1.0 & 1.0 & $6.5 \times 10^{8}$ \\
1.0 & 2.0 & $7.6 \times 10^{5}$ \\
1.0 & 4.0 & $2.4 \times 10^{4}$ \\
2.0 & 1.0 & 0.82 \\
2.0 & 2.0 & 0.71 \\
2.0 & 4.0 & 0.49 \\
4.0 & 1.0 & 0.45 \\
4.0 & 2.0 & 0.42 \\
4.0 & 4.0 & 0.38 \\
\hline
\end{tabular}

Based on data from Fig. 2A, CI values were calculated using the CI-isobologram method of Chou and Talalay (13).

initially monitored cell death by flow cytometric analysis of PI uptake. After 1-h treatment with SAHA (1-4 $\mu \mathrm{M})$ or NaB (2-6 mM), A2780 cells were exposed to aspirin (1-4 mM) for another $48 \mathrm{~h}$. As presented in Fig. 1A, non-pretreated cells were marginally sensitive to aspirin under these conditions. However, when cells were pre-exposed to HDIs, aspirin evoked cell death in a concentration-dependent manner. For example, in the presence of $2 \mu \mathrm{M}$ SAHA, which was not cytotoxic by itself, treatment with aspirin caused cell death in $\leq 29.4 \%$ of cells. We tested the combination of HDIs and aspirin for synergy by the CI-isobologram method $(\mathrm{CI}<0.9$ is indicative for a synergistic interaction) (13). This analysis revealed clear synergy for all combinations involving 2 and $4 \mu \mathrm{M}$ SAHA (CI, 0.07-0.63; Table I) as well as 4 and $6 \mathrm{mM}$ $\mathrm{NaB}$ (CI, 0.03-0.58; Table II).

HDIs as well as aspirin have been shown to induce cell death by affecting the mitochondria $(14,15)$. Thus, in order to verify the observed cooperative interaction of HDIs and aspirin by another read-out, we determined $\Delta \psi_{\mathrm{m}}$. We found that aspirin as a single agent had little effect on mitochondria (Fig. 1B). Likewise, SAHA or NaB applied alone resulted in decay of $\Delta \psi_{\mathrm{m}}$ in maximally $18.4 \%$ of cells. However, in the combined drug group, we observed $\Delta \psi_{\mathrm{m}}$ dissipation in up to $74.2 \%$ of cells.

We employed Western blot analysis to examine the effect of HDIs and aspirin on the acetylation status of histone $\mathrm{H} 3$. After 1-h treatment with SAHA, A2780 cells were exposed to aspirin for $24 \mathrm{~h}$, and histone $\mathrm{H} 3$ acetylation was analyzed by an acetylated $\mathrm{H} 3$-specific antibody. As demonstrated in Fig. 1C, aspirin had no effect on histone acetylation, neither in the absence nor presence of SAHA, while SAHA and $\mathrm{NaB}$ induced histone H3 hyperacetylation. Different incubation times with aspirin (1-48 h) did not result in increased histone acetylation (not shown).

Effect of histone deacetylase inhibitors in combination with salicylic acid, SC-560, or NS-398. To investigate whether the synergistic action of HDIs and aspirin was due to the acetyl 
A

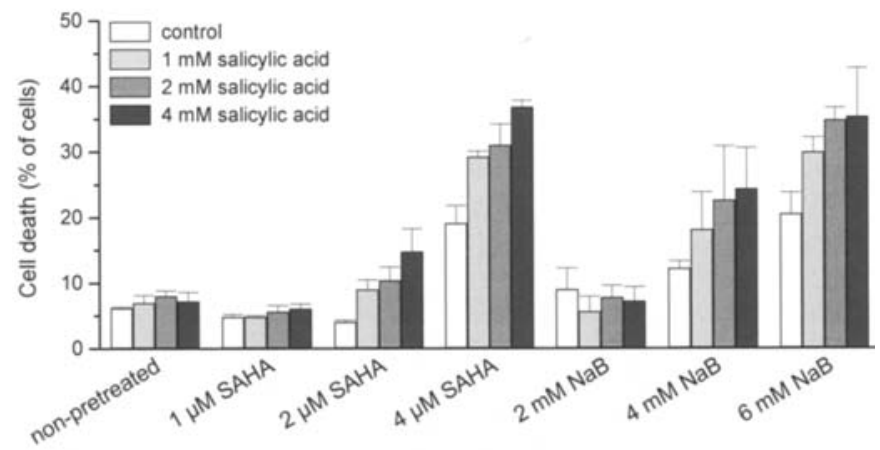

B

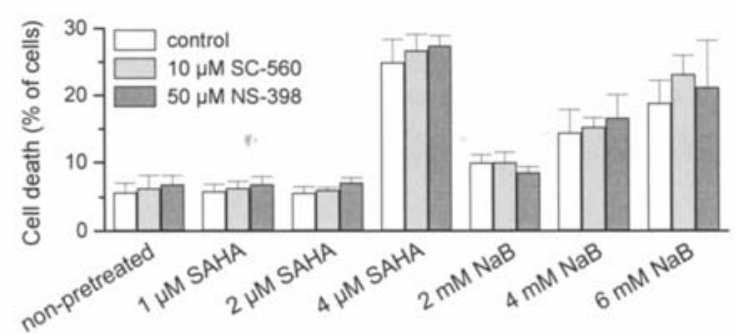

Figure 2. Induction of cell death in A2780 cells by HDIs and salicylic acid, SC-560, or NS-398. After 1-h treatment with HDIs, cells were exposed to (A) salicylic acid or (B) SC-560 or NS-398 for another 48 h. Cell death was determined by flow cytometric analysis of PI uptake. Means \pm SD of each three separate experiments are shown.

or the salicyl moiety of aspirin, we combined SAHA and $\mathrm{NaB}$ with salicylic acid, the deacetylated metabolite of aspirin. Fig. 2A shows that salicylic acid alone was hardly toxic to A2780 cells. Low doses of HDIs ( $1 \mu \mathrm{M}$ SAHA, $2 \mathrm{mM} \mathrm{NaB})$, which already sensitized cells to aspirin (Fig. 1A), had no effect on the susceptibility to salicylic acid. However, in conjunction with higher concentrations of HDIs, salicylic acid elicited cell death in a dose-dependent manner. These data were tested for synergy by the CI-isobologram method. The calculated CI values (0.24-0.82) indicated a synergistic to weakly synergistic effect (Tables III and IV). In order to investigate whether the synergistic induction of cell death by HDIs and aspirin involved the inhibition of COX activity, we employed SC-560 and NS-398, selective inhibitors of COX-1 and -2, respectively. As demonstrated in Fig. 2B, SC-560 and NS-398 were hardly cytotoxic in A2780 cells when applied alone or in conjunction with HDIs.

\section{Discussion}

This study was driven by the hypothesis that HDIs and aspirin might interact cooperatively to induce cell death and increase histone acetylation in cancer cells. We found that the HDIs SAHA and $\mathrm{NaB}$ cooperated with aspirin in exerting a cytotoxic effect on A2780 ovarian cancer cells, as judged by flow cytometric analyses of PI uptake and $\Delta \psi_{\mathrm{m}}$. The CIisobologram analysis provided evidence that the interaction between HDIs and aspirin was truly synergistic. However, contrary to our expectations, we did not observe a combinatorial action of HDIs and aspirin on histone acetylation.
Table IV. Combination index values for sodium butyrate plus salicylic acid.

\begin{tabular}{lcc}
\hline $\mathrm{NaB}(\mathrm{mM})$ & Salicylic acid $(\mathrm{mM})$ & $\mathrm{CI}$ \\
\hline 2.0 & 1.0 & $3.8 \times 10^{5}$ \\
2.0 & 2.0 & 1.69 \\
2.0 & 4.0 & 9.02 \\
4.0 & 1.0 & 0.68 \\
4.0 & 2.0 & 0.43 \\
4.0 & 4.0 & 0.37 \\
6.0 & 1.0 & 0.35 \\
6.0 & 2.0 & 0.24 \\
6.0 & 4.0 & 0.24
\end{tabular}

Based on data from Fig. 2A, CI values were calculated using the CI-isobologram method of Chou and Talalay (13).

Treatment with aspirin had no effect on the acetylation status of histones, neither in the presence nor absence of HDIs (while SAHA and $\mathrm{NaB}$ induced pronounced histone hyperacetylation). It can therefore be concluded that the synergistic cytotoxic action of HDIs and aspirin did not arise from a cooperative induction of histone acetylation. However, in addition to histones, many non-histone proteins are modified by acetylation and, thus, are potential targets of HDIs (16). For example, the acetylation of p53 has been shown to stimulate its DNA-binding activity (17). Therefore, we cannot exclude the possibility that HDIs and aspirin may cooperate in inducing hyperacetylation of non-histone proteins.

In order to gain insight into the mode of action of the combination treatment with HDIs and aspirin, we employed salicylic acid, the deacetylated metabolite of aspirin. If the combination of HDIs with salicylic acid was as efficient as the combination of HDIs with aspirin, an acetyl groupindependent action of aspirin could be concluded. Vice versa, a lesser efficacy of the combination of HDIs with salicylic acid would indicate an acetyl group-dependent action. Our results suggest that both the acetyl and the salicyl moiety contribute to aspirin's synergistic interaction with HDIs. On the one hand, we noted a cooperative effect of HDIs with salicylic acid, and CI-isobologram analysis indicated that it was synergistic. On the other hand, the combination of HDIs and salicylic acid was less potent than the combination of HDIs and aspirin; in particular, low concentrations of SAHA or $\mathrm{NaB}$ sensitized cells to aspirin but not to salicylic acid.

Aspirin is supposed to function primarily by inhibiting COX activity. Two COX isoforms have been described: COX-1 is constitutively expressed in many tissues, while COX-2 is inducible by diverse stimuli, such as growth factors, cytokines, and mitogens (7). In the majority of studies, the antineoplastic effects of NSAIDs including aspirin have been attributed to the inhibition of COX-2 $(18,19)$. However, Daikoku et al have reported that the COX-1-selective inhibitor SC-560 reduced tumor growth in a mouse model of ovarian carcinoma, suggesting that COX-1 may also be a target for 
cancer treatment (20). To find out whether the cooperative action of HDIs and aspirin involved the inhibition of COX activity, we studied the effect of SC-560 and the COX-2selective inhibitor NS-398 in combination with HDIs. The COX inhibitors had little effect when applied alone or in conjunction with SAHA or $\mathrm{NaB}$, arguing against the possibility that the combinatorial effect of HDIs and aspirin stemmed from the inhibition of COX-1 or -2 . In concordance, in a recent assessment of aspirin and non-aspirin NSAID use in postmenopausal women, only aspirin use was found to be associated with lower cancer incidence and mortality (21).

Therefore, which mechanism may account for the synergistic interaction of HDIs and aspirin? Since our results militate against a combination effect on histone acetylation or COX activity, we can presently offer only some speculative explanations. For example, HDIs have been shown to cooperate with the proteasome inhibitor bortezomib (22), and aspirin has been reported to inhibit proteasome function (23). Likewise, HDI-induced cell death can be augmented by inhibiting the antiapoptotic transcription factor $\mathrm{NF}-\kappa \mathrm{B}$ $(24,25)$, whose activation is blocked by aspirin and salicylic acid $(26,27)$. It is currently under discussion that salicylates and other NSAIDs in general may have synergistic efficacy when combined with anticancer agents $(28,29)$. However, there are also a number of studies that suggest otherwise. For example, it has been shown that aspirin can induce resistance against doxorubicin in prostate cancer cells (30) and that it can antagonize the apoptotic response of colon cancer cells to etoposide and irinotecan (31). NS-398 has been reported to protect ovarian cancer cells against paclitaxel (32) and another COX-2-selective inhibitor, nimesulide, has been found to inhibit the cytotoxic effect of cisplatin in head and neck cancer cells (33). Therefore, a potential benefit of adding NSAIDs to anticancer chemotherapy may depend on the tumor entity and/ or the drug applied and must be unambiguously established. Our study demonstrates that HDIs and aspirin interact favorably in A2780 ovarian cancer cells and, hence, suggests that the combination of HDIs and aspirin warrants a more in-depth exploration for the treatment of cancer.

\section{Acknowledgements}

We thank J. Gänge and A. Plath for their excellent technical assistance. This study was supported by the 'Wilhelm SanderStiftung, Neustadt/Donau'.

\section{References}

1. Bolden JE, Peart MJ and Johnstone RW: Anticancer activities of histone deacetylase inhibitors. Nat Rev Drug Discov 5: 769-784, 2006.

2. Kouraklis $\mathrm{G}$ and Theocharis $\mathrm{S}$ : Histone deacetylase inhibitors: A novel target of anticancer therapy (Review). Oncol Rep 15: 489-494, 2006.

3. Thun MJ, Henley SJ and Patrono C: Nonsteroidal antiinflammatory drugs as anticancer agents: mechanistic, pharmacologic, and clinical issues. J Natl Cancer Inst 94: 252-266, 2002.

4. Ulrich CM, Bigler J and Potter JD: Non-steroidal antiinflammatory drugs for cancer prevention: promise, perils and pharmacogenetics. Nat Rev Cancer 6: 130-140, 2006.
5. Flossmann E, Rothwell PM and on behalf of the British Doctors Aspirin Trial and the UK-TIA Aspirin Trial: Effect of aspirin on long-term risk of colorectal cancer: consistent evidence from randomised and observational studies. Lancet 369: 1603-1613, 2007.

6. Schildkraut JM, Moorman PG, Halabi S, Calingaert B, Marks JR and Berchuck A: Analgesic drug use and risk of ovarian cancer. Epidemiology 17: 104-107, 2006.

7. Smith WL, DeWitt DL and Garavito RM: Cyclooxygenases: structural, cellular, and molecular biology. Annu Rev Biochem 69: 145-182, 2000.

8. Tegeder I, Pfeilschifter J and Geisslinger G: Cyclooxygenaseindependent actions of cyclooxygenase inhibitors. FASEB J 15 : 2057-2072, 2001.

9. Pinckard RN, Hawkins D and Farr RS: In vitro acetylation of plasma proteins, enzymes and DNA by aspirin. Nature 219: 68-69, 1968.

10. Rodriguez-Burford C, Barnes MN, Oelschlager DK, Myers RB, Talley LI, Partridge EE and Grizzle WE: Effects of nonsteroidal anti-inflammatory agents (NSAIDs) on ovarian carcinoma cell lines: preclinical evaluation of NSAIDs as chemopreventive agents. Clin Cancer Res 8: 202-209, 2002.

11. Cooper AL, Greenberg VL, Lancaster PS, van Nagell JR Jr, Zimmer SG and Modesitt SC: In vitro and in vivo histone deacetylase inhibitor therapy with suberoylanilide hydroxamic acid (SAHA) and paclitaxel in ovarian cancer. Gynecol Oncol 104: 596-601, 2007.

12. Sonnemann J, Gange J, Pilz S, Stotzer C, Ohlinger R, Belau A, Lorenz $\mathrm{G}$ and Beck JF: Comparative evaluation of the treatment efficacy of suberoylanilide hydroxamic acid (SAHA) and paclitaxel in ovarian cancer cell lines and primary ovarian cancer cells from patients. BMC Cancer 6: 183, 2006.

13. Chou TC: Theoretical basis, experimental design, and computerized simulation of synergism and antagonism in drug combination studies. Pharmacol Rev 58: 621-681, 2006.

14. Peart MJ, Tainton KM, Ruefli AA, Dear AE, Sedelies KA, O'Reilly LA, Waterhouse NJ, Trapani JA and Johnstone RW: Novel mechanisms of apoptosis induced by histone deacetylase inhibitors. Cancer Res 63: 4460-4471, 2003

15. Pique M, Barragan M, Dalmau M, Bellosillo B, Pons G and Gil J: Aspirin induces apoptosis through mitochondrial cytochrome c release. FEBS Lett 480: 193-196, 2000.

16. Drummond DC, Noble CO, Kirpotin DB, Guo Z, Scott GK and Benz CC: Clinical development of histone deacetylase inhibitors as anticancer agents. Annu Rev Pharmacol Toxicol 45: 495-528, 2005.

17. $\mathrm{Gu} \mathrm{W}$ and Roeder RG: Activation of p53 sequence-specific DNA binding by acetylation of the p53 C-terminal domain. Cell 90: 595-606, 1997.

18. Dannenberg AJ and Subbaramaiah K: Targeting cyclooxygenase- 2 in human neoplasia: rationale and promise. Cancer Cell 4: 431-436, 2003.

19. Brown JR and Dubois RN: COX-2: a molecular target for colorectal cancer prevention. J Clin Oncol 23: 2840-2855, 2005.

20. Daikoku T, Wang D, Tranguch S, Morrow JD, Orsulic S, Dubois RN and Dey SK: Cyclooxygenase-1 is a potential target for prevention and treatment of ovarian epithelial cancer. Cancer Res 65: 3735-3744, 2005.

21. Bardia A, Ebbert JO, Vierkant RA, Limburg PJ, Anderson K, Wang AH, Olson JE, Vachon CM and Cerhan JR: Association of aspirin and nonaspirin nonsteroidal anti-inflammatory drugs with cancer incidence and mortality. J Natl Cancer Inst 99: 881-889, 2007.

22. Yu C, Rahmani M, Conrad D, Subler M, Dent P and Grant S: The proteasome inhibitor bortezomib interacts synergistically with histone deacetylase inhibitors to induce apoptosis in $\mathrm{Bcr} / \mathrm{Abl}^{+}$cells sensitive and resistant to STI571. Blood 102: 3765-3774, 2003 .

23. Dikshit P, Chatterjee M, Goswami A, Mishra A and Jana NR: Aspirin induces apoptosis through the inhibition of proteasome function. J Biol Chem 281: 29228-29235, 2006.

24. Rundall BK, Denlinger CE and Jones DR: Combined histone deacetylase and NF-kappaB inhibition sensitizes non-small cell lung cancer to cell death. Surgery 136: 416-425, 2004.

25. Sonnemann J, Hartwig M, Plath A, Saravana KK, Muller C and Beck JF: Histone deacetylase inhibitors require caspase activity to induce apoptosis in lung and prostate carcinoma cells. Cancer Lett 232: 148-160, 2006.

26. Kopp E and Ghosh S: Inhibition of NF-kappa B by sodium salicylate and aspirin. Science 265: 956-959, 1994. 
27. Grilli M, Pizzi M, Memo M and Spano P: Neuroprotection by aspirin and sodium salicylate through blockade of NF-kappaB activation. Science 274: 1383-1385, 1996.

28. McCarty MF and Block KI: Preadministration of high-dose salicylates, suppressors of NF-kappaB activation, may increase the chemosensitivity of many cancers: an example of proapoptotic signal modulation therapy. Integr Cancer Ther 5: 252-268, 2006.

29. de Groot DJ, de Vries EG, Groen HJ and de Jong S: Nonsteroidal anti-inflammatory drugs to potentiate chemotherapy effects: from lab to clinic. Crit Rev Oncol Hematol 61: 52-69, 2007.

30. Rotem R, Tzivony Y and Flescher E: Contrasting effects of aspirin on prostate cancer cells: suppression of proliferation and induction of drug resistance. Prostate 42: 172-180, 2000.
31. Ricchi P, Di Matola T, Ruggiero G, Zanzi D, Apicella A, di Palma A, Pensabene M, Pignata S, Zarrilli R and Acquaviva AM: Effect of non-steroidal anti-inflammatory drugs on colon carcinoma Caco-2 cell responsiveness to topoisomerase inhibitor drugs. Br J Cancer 86: 1501-1509, 2002.

32. Munkarah AR, Genhai Z, Morris R, Baker VV, Deppe G, Diamond MP and Saed GM: Inhibition of paclitaxel-induced apoptosis by the specific COX-2 inhibitor, NS398, in epithelial ovarian cancer cells. Gynecol Oncol 88: 429-433, 2003.

33. Czembirek C, Eder-Czembirek C, Erovic BM, Turhani D, Selzer E and Thurnher D: Inhibition of cytotoxicity of cisplatin by cyclooxygenase-2 inhibitor nimesulide in head and neck cancer cell lines. Oncol Rep 14: 1523-1526, 2005. 